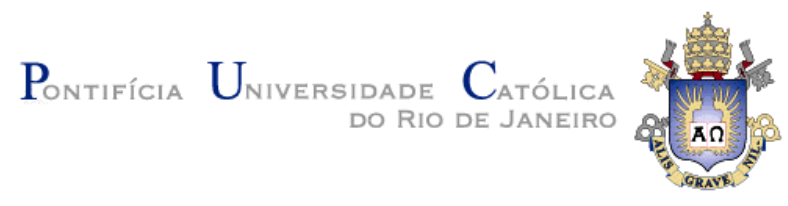

Marcelle Pereira Mota

\title{
PoliFacets: um modelo de design da metacomunicação de documentos ativos para apoiar o ensino e aprendizado de programação
}

Tese apresentada ao Programa de Pós-graduação em Informática do Departamento de Informática do Centro Técnico e Científico da PUC-Rio, como requisito parcial para a obtenção do grau de Doutor em Informática.

Orientador: Prof ${ }^{a}$. Clarisse Sieckenius de Souza 
Marcelle Pereira Mota

\title{
Polifacets: um modelo de design da metacomunicação de documentos ativos para apoiar o ensino e aprendizado de programação
}

Tese apresentada ao Programa de Pós-graduação em Informática do Departamento de Informática do Centro Técnico e Científico da PUC-Rio, como requisito parcial para a obtenção do grau de Doutor em Informática.

\author{
Profa. Clarisse Sieckenius de Souza \\ Orientador \\ Departamento de Informática - PUC-Rio
}

Prof ${ }^{a}$. Milene Selbach Silveira

PUC-RS

Profa. Tayana Uchôa Conte

UFAM

Prof ${ }^{a}$. Simone Diniz Junqueira Barbosa

Departamento de Informática - PUC-Rio

Prof. Alberto Barbosa Raposo

Departamento de Informática - PUC-Rio

Prof. José Eugenio Leal

Coordenador Setorial do Centro Técnico Científico - PUC-Rio 
Todos os direitos reservados. É proibida a reprodução total ou parcial do trabalho sem autorização da universidade, da autora e do orientador.

Marcelle Pereira Mota

Graduou-se em Bacharelado em Ciência da Computação pela Universidade Federal do Pará (2007). Possui mestrado em Ciência da Computação na área de Informática na educação pela Universidade Federal do Pará (2010).

Ficha Catalográfica

Mota, Marcelle Pereira

PoliFacets : um modelo de design da metacomunicação de documentos ativos para apoiar o ensino e aprendizado de programação / Marcelle Pereira Mota ; orientador: Clarisse Sieckenius de Souza. - 2014.

202 f. : Il. (color.) ; $30 \mathrm{~cm}$

Tese (doutorado)-Pontifícia Universidade Católica do Rio de Janeiro, Departamento de Informática, 2014.

Inclui bibliografia

1. Informática - Teses. 2. PoliFacets. 3. Documentação ativa. 4. Raciocínio computacional. 5. Engenharia semiótica. I. Souza, Clarisse Sieckenius de. II. Pontifícia Universidade Católica do Rio de Janeiro. Departamento de Informática. III. Título.

CDD: 004 
Aos meus pais, Adonai e Maura. 


\section{Agradecimentos}

Agradeço a Deus pela saúde e paz. Agradeço à minha família. Meus pais, Adonai e Maura, obrigada por estarem sempre ao meu lado e acreditar que eu sou capaz de realizar meus sonhos. Aos meus irmãos e cunhadas, Marcelo e Silvana, Marcel e Ariane, agradeço pela torcida e apoio diante dos obstáculos. Às minhas afilhadas, Yasmin e Julia, agradeço por entenderem a minha ausência.

Agradeço à professora Clarisse pela orientação, pelo tempo dedicado, pelo incentivo nos momentos difíceis e pela confiança. Obrigada por me ensinar como é ser uma pesquisadora, orientadora e professora através do seu exemplo.

Agradeço aos amigos que estiveram a poucos cliques de distância: Lucélia, Murilo, Eder, Marcelo, Alline, Hugo e Suanne. Em especial, agradeço a Gaby e a Luana por acreditarem na minha capacidade até quando eu mesmo duvido. Agradeço ao Eduardo pela companhia durante um período importante do curso e por compartilhar os mesmos sonhos. Agradeço à Tânia, in memoriam, por ter sido minha professora desde a infância e por ter estado presente em todos os momentos importantes, mas agora estará somente em meu coração.

Agradeço aos amigos da CCPA que fizeram parte da minha trajetória: Tomás, Carla Leitão, Carla Ourofino, Patrícia, Luzzia, Victor e Rustam. Ao Tomás, agradeço pela oportunidade de trabalho e aprendizado. Agradeço a vocês pela amizade, pelas longas conversas, pelos momentos de distração, pelo incentivo e pelo apoio incondicional diante do meu objetivo. Agradeço aos companheiros do SERG pelas contribuições: Carla, Luciana, Ingrid, Juliana, Cleyton, João, Eduardo, Hugo, Martha, Rosana, Cátia, Luiz, José Antônio, Bruno, Silvia, Raquel, Simone e Milene. Em especial, agradeço à Ingrid pela amizade e atenção de sempre. Agradeço à Carla Leitão, Nelson e Sofia pelo carinho com que me receberam em sua casa, pela torcida, pelo incentivo e pela amizade.

Por fim, agradeço aos professores do DI da PUC-Rio que contribuíram para minha formação e a todas as pessoas que ajudaram direta ou indiretamente. Além disso, agradeço ao incentivo financeiro do DI, da PUC-Rio, da CAPES e do CNPq. 


\section{Resumo}

Mota, Marcelle Pereira; de Souza, Clarisse Sieckenius. PoliFacets: um modelo de design da metacomunicação de documentos ativos para apoiar o ensino e aprendizado de programação. Rio de Janeiro, 2014. 202p. Tese de Doutorado - Departamento de Informática, Pontifícia Universidade Católica do Rio de Janeiro.

Atualmente há uma necessidade de uso da tecnologia para efetivar a participação do cidadão na sociedade. Os usuários estão deixando de ser somente consumidores passivos de software e uma fração crescente deles já passa a usar a tecnologia como um meio de expressão de novas ideias e oportunidades. Em um cenário democrático de futuro, quantas mais pessoas puderem manifestar-se através do uso eficaz e eficiente de tecnologia, menor o risco de que aqueles que podem fazê-lo determinem o que os demais poderão fazer. Porém, o processo de ensino e aprendizado de 'raciocínio computacional', base de competência para a autoexpressão através de software, é um grande desafio. Os professores de escolas de ensino fundamental e médio, por exemplo, geralmente não têm formação ou auxílio para ensinar conteúdos que envolvem conceitos de computação com os quais não estão familiarizados e, portanto, precisam, eles próprios, aprender. Esta tese apresenta um modelo para o design de documentação ativa destinada a apoiar o ensino e aprendizado de raciocínio computacional. O modelo é baseado em Engenharia Semiótica e sua instanciação em um cenário real de uso resultou em um documento utilizado em vários estudos empíricos realizados ao longo de três anos, em escolas de ensino fundamental e médio na região metropolitana do Rio de Janeiro. Na dimensão técnico-científica, a principal contribuição da tese é uma ferramenta epistêmica para estruturar as análises e decisões durante o design da metacomunicação de documentos ativos destinados a apoiar o ensino e aprendizado de autoexpressão através de software.

\section{Palavras-chave}

PoliFacets; documentação ativa; raciocínio computacional; Engenharia Semiótica. 


\section{Abstract}

Mota, Marcelle Pereira; de Souza, Clarisse Sieckenius (advisor). PoliFacets: a design model for the metacommunication of active documents to support teaching and learning of computer programming. Rio de Janeiro, 2014. 202p. DSc Thesis - Departamento de Informática, Pontifícia Universidade Católica do Rio de Janeiro.

Nowadays, there is a need to use technology to effect citizen participation in society. Users are no longer only passive software consumers and a growing share of them are using technology as a medium to express new ideas and opportunities. In a democratic future scenario, the more people can manifest themselves through the effective and efficient use of technology, the lower the risk that those who can do it determine what others will do. However, the process of teaching and learning 'computational thinking', which is the basic skill for self-expression through software, is a big challenge. Teachers need to learn computational concepts themselves before they can teach them to students. In elementary and high school they generally do not have support for teaching this kind of content. This thesis presents a model for the design of active documentation which aims at supporting the teaching and learning of computational thinking. The model is based on Semiotic Engineering theory and its instantiation in a real scenario came about as an active document used in several empirical studies during three years with elementary and high schools in the metropolitan region of Rio de Janeiro. Technically and scientifically, the main contribution of this thesis is an epistemic tool for structure analyses and decisions during the design of metacommunication of active documents to support the teaching and learning of self-expression through software.

\section{Keywords}

PoliFacets; active documentation; computational thinking; Semiotic Engineering. 


\section{Sumário}

1 Introdução 16

1.1. Contexto da pesquisa 16

1.2. Metodologia 28

1.3. Organização do texto 31

2 Trabalhos relacionados 32

2.1. Definições e abordagens do ensino e aprendizado de Raciocínio computacional 32

2.2. Tecnologias de apoio e colaboração 34

2.2.1. Ambientes de programação, compartilhamento de recursos e troca de experiências $\quad 35$

2.2.2. Ferramentas de auxílio à compreensão e depuração de programas

2.2.3. Documentação ativa 43

2.3. Software como forma de participação social 45

2.4. Relações dos trabalhos citados com a pesquisa 48

30 modelo PoliFacets 54

3.1. Conceitos de Engenharia Semiótica 54

3.2. Estudos empíricos 65

3.2.1. Estudo inicial 67

3.2.2. I workshop para professores $\quad 70$

3.2.3. Regras e narrativas de programas 77

3.2.4. Expressão de conexões entre agentes 79

3.2.5. II workshop para professores 87

3.2.6. Estratégias de comunicação 95

3.3. Elaboração teórica do modelo 101

3.4. Componentes estruturais e suas relações 103

3.5. Ontologia de metacomunicação 105

3.5.1. Perspectivas do usuário e do sistema 105 
3.5.2. Perspectiva da interação

3.6. Visão completa do modelo

4 A instância PoliFacets-AS

4.1. Facetas instanciadas e suas ligações

4.1.1. Faceta 'descrição'

122

4.1.2. Faceta 'na prática'

4.1.3. Faceta 'planilhas'

125

4.1.4. Faceta 'regras'

128

4.1.5. Faceta 'tags'

129

4.1.6. Faceta 'conexões'

4.2. Facetas idealizadas e suas ligações

133

4.2.1. Faceta $A$

135

4.2.2. Faceta $B$

135

4.2.3. Faceta $\mathrm{C}$

136

4.2.4. Faceta $D$

137

4.2.5. Faceta $E$

139

4.2.6. Faceta $F$

139

4.2.7. Faceta $G$

140

4.2.8. Faceta $\mathrm{H}$

141

4.2.9. Faceta I

141

4.2.10. Faceta J

142

4.3. Avaliação

143

4.3.1. Recepção das facetas

143

4.3.2. Validação dos resultados

149

5 Considerações finais

162

5.1. Lições aprendidas

163

5.2. Limitações conhecidas

164

5.3. Contribuições da pesquisa

166

5.4. Trabalhos futuros

167

5.4.1. Pesquisas de curto prazo

168

5.4.2. Pesquisas de médio prazo

168

5.4.3. Pesquisas de longo prazo 
Referências

$\begin{array}{ll}\text { Apêndices } & 180\end{array}$

Material do estudo 'Regras e narrativas de programas' 180

Material do estudo 'Expressão de conexões entre agentes' 181

Questionário A do 'Il workshop para professores' 183

Questionário B do 'Il workshop para professores' 184

Scripts do estudo 'Recepção das facetas' 185

$\begin{array}{ll}\text { Termos de consentimento } & 187\end{array}$

$\begin{array}{ll}\text { Estudo inicial } & 187\end{array}$

Expressão de conexões entre agentes 189

Estratégias de comunicação 191

Validação dos resultados 192

$\begin{array}{ll}\text { Anexos } & 193\end{array}$

Questionário A do 'I workshop para professores’ 193

Questionário B do 'I workshop para professores' 193

Questionário do estudo 'Recepção das facetas' 195

$\begin{array}{ll}\text { Termos de consentimento } & 195\end{array}$

I workshop de professores 195

$\begin{array}{ll}\text { Regras e narrativas de programas } & 197\end{array}$

Il workshop de professores 199

$\begin{array}{ll}\text { Recepção das facetas } & 201\end{array}$ 


\section{Lista de figuras}

Figura 1.1 - Construção da simulação 'produção de mel' no AS 22

Figura 1.2 - Faceta 'planilha' da simulação 'produção de mel' 25

Figura 1.3 - Conexões do agente flor_2 25

Figura 1.4 - Conexões do agente abelha_2 26

Figura 1.5 - Etapas para a construção do Modelo PoliFacets 29

Figura 2.1 - Grafo de padrões de raciocínio computacional 41

Figura 3.1 - Esquema geral de metacomunicação em IHC 57

Figura 3.2 - Exemplo de paráfrase no AS 62

Figura 3.3 - Exemplo de figura de linguagem no AS 63

Figura 3.4 - Exemplo de Ressignificação no AS 64

Figura 3.5 - Projeto Pacman simplificado $\quad 67$

Figura 3.6 - Alguns jogos do primeiro workshop 74

Figura 3.7 - Simulação de agentes fora da janela de visualização $\quad 75$

Figura 3.8 - Lista de agentes na planilha do projeto Apartamentos $\quad 76$

Figura 3.9 - Relações do agente A com outros agentes 80

Figura 3.10 - Agentes da simulação 81

Figura 3.11 - Conexões do agente 'a' com outros agentes 83

Figura 3.12 - Progressão de acordo com as perspectivas culturais $\quad 88$

Figura 3.13 - Projeto do participante P7 93

Figura 3.14 - Jogo do Participante P5 94

Figura 3.15 - Jogo do Participante P6 94

Figura 3.16 - Cabeçalho das facetas do projeto de P6 95

Figura 3.17 - SideTalk durante a navegação no PoliFacets 96

Figura 3.18 - Modelo PoliFacets no contexto de design da tecnologia 104

Figura 3.19 - Modelo PoliFacets 114

Figura 4.1 - Modelo PoliFacets-AS 118

Figura 4.2 - Distribuição das facetas 120

Figura 4.3 - Faceta 'descrição' do Pacman 122

Figura 4.4 - Faceta 'na prática' do projeto Frogger 124

Figura 4.5 - Faceta 'planilhas' do Frogger 125 
Figura 4.6 - Opções de visualização da planilha 126

Figura 4.7 - Grade com os agentes river, ground e street escondidos 126

Figura 4.8 - Regras de comportamento do Frogger 128

Figura 4.9 - Linguagem pseudonatural e Linguagem de código do AS 129

Figura 4.10 - Tags do Frogger 130

Figura 4.11 - Tags do Pacman $\quad 130$

Figura 4.12 - Diagrama de conexões do agente frog 131

Figura 4.13 - Diagrama de conexões do agente 'log' 132

Figura 4.14 - Planilha do jogo Frogger modificada 136

Figura 4.15 - llustração da faceta de transição de planilhas 138

Figura 4.16 - Visão textual no NCL Composer 150

Figura 4.17 - Visão estrutural no NCL Composer 151

Figura 4.18 - Visão de layout no NCL Composer 151

Figura 4.19 - Modelo PoliFacets-NCL 152

Figura 4.20 - Faceta 'descrição' no PoliFacets-NCL 154

Figura 4.21 - Faceta 'código' no PoliFacets-NCL 155

Figura 4.22 - Faceta 'estrutura' no PoliFacets-NCL 156

Figura 4.23 - Faceta 'layout' no PoliFacets-NCL 157

Figura 4.24 - Faceta 'na prática' no PoliFacets-NCL 158 


\section{Lista de quadros}

Quadro 1.1 - Efeitos visuais no tempo 24

Quadro 3.1 - Cronograma do primeiro workshop 71

Quadro 3.2 - Participantes do primeiro workshop 72

Quadro 3.3 - Organização dos participantes 82

Quadro 3.4 - Cronograma do segundo workshop 87

Quadro 3.5 - Planejamento de acordo com as metáforas 89

Quadro 3.6 - Participantes do segundo workshop 90

Quadro 3.7 - Participantes 97

Quadro 3.8 - Exemplo de figuras de linguagem com reformulação 109

Quadro 3.9 - Exemplo de expansão linguística com reescrita 111

$\begin{array}{ll}\text { Quadro } 4.1 \text { - Cenário do estudo } & 144\end{array}$ 


\section{Lista de tabelas}

Tabela 1.1 - Esquema de interação entre os agentes e seus efeitos 23

Tabela 3.1 - Operações simbólicas na perspectiva do sistema $\quad 59$

Tabela 3.2 - Operações semióticas na perspectiva do usuário 61

Tabela 3.3 - Organização dos estudos entre as fases da pesquisa $\quad 66$

Tabela 3.4 - Estudos e resumo da contribuição para a construção do modelo 102

Tabela 3.5 - Matriz com as perspectivas do usuário e do sistema 106

Tabela 3.6 - Perspectiva da interação 112

Tabela 3.7 - Grade da ontologia de metacomunicação 116

Tabela 4.1 - Grade da ontologia de metacomunicação no PoliFacets-AS

Tabela 4.2 - Grade da ontologia de metacomunicação no PoliFacets-AS com facetas idealizadas $\quad 134$

Tabela 4.3 - Facetas e ordem de visualização 145

Tabela 4.4 - Grade da ontologia de metacomunicação no PoliFacets-NCL 
"Sonhos são gratuitos.

Transformá-los em realidade tem um preço."

E. J. Gibs

"Logic will get you from A to B.

Imagination will take you everywhere."

Albert Einstein 